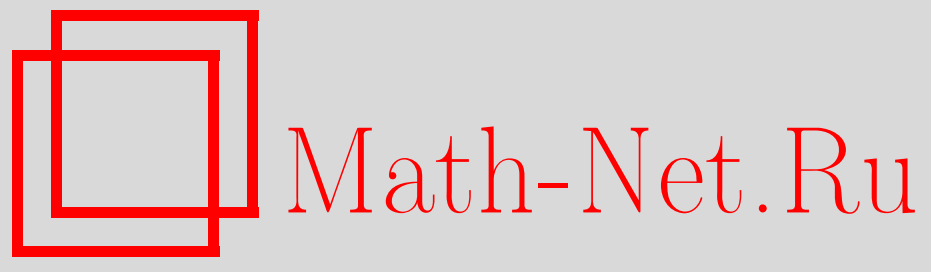

И. В. Вьюгин, Фуксовы системы с вполне приводимой монодромией, Матем. заметки, 2009, том 85, выпуск $6,817-825$

DOI: https://doi.org/10.4213/mzm7660

Использование Общероссийского математического портала Math-Net.Ru подразумевает, что вы прочитали и согласны с пользовательским соглашением http://www . mathnet.ru/rus/agreement

Параметры загрузки:

IP: 18.209 .158 .208

26 апреля 2023 г., 18:05:20

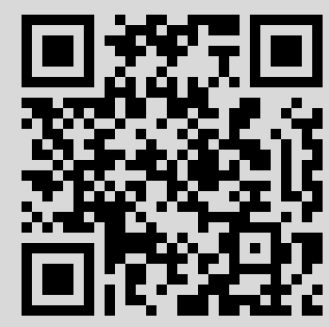


Том 85 выпуск 6 июнь 2009

УДК 517

\section{Фуксовы системы с вполне приводимой монодромией}

\section{И. В. Вьюгин}

В работе исследованы вопросы положительной разрешимости проблемы Римана-Гильберта для представлений, имеющих вид прямой суммы $\chi=\chi_{1} \oplus \chi_{2}$. Доказано, что любое представление $\chi_{1}$ может быть реализовано как прямое слагаемое в представлении $\chi$ монодромии фуксовой системы. Получены и другие результаты, на основе которых представлен простой метод построения контрпримеров к проблеме Римана-Гильберта.

Библиография: 5 названий.

1. Введение. Рассмотрим систему линейных дифференциальных уравнений

$$
\frac{d y}{d z}=B(z) y, \quad y(z) \in \mathbb{C}^{p}
$$

из $p$ уравнений с мероморфной на сфере Римана матрицей $B(z)$, голоморфной вне точек $a_{1}, \ldots, a_{n}$.

Представлением монодромии или монодромией данной системы уравнений называется представление

$$
\chi: \pi_{1}\left(\overline{\mathbb{C}} \backslash\left\{a_{1}, \ldots, a_{n}\right\}, z_{0}\right) \rightarrow G L(p, \mathbb{C})
$$

фундаментальной группы пространства $\overline{\mathbb{C}} \backslash\left\{a_{1}, \ldots, a_{n}\right\}$ в пространство невырожденных комплексных матриц порядка $p$, которое определяется следующим образом. В окрестности неособой точки $z_{0}$ рассмотрим фундаментальную матрицу $Y(z)$ решений системы (1). При аналитическом продолжении функций $Y(z)$ вдоль произвольной петли $\gamma$, начинающейся в точке $z_{0}$ и лежащей в $\overline{\mathbb{C}} \backslash\left\{a_{1}, \ldots, a_{n}\right\}$, фундаментальная матрица $Y(z)$ переходит в (вообще говоря, другую) фундаментальную матрицу $\tilde{Y}(z)$. Два базиса связаны с помощью невырожденной матрицы перехода $G_{\gamma}$, соответствующей петле $\gamma$ :

$$
Y(z)=\tilde{Y}(z) G_{\gamma}
$$

Отображение $[\gamma] \mapsto G_{\gamma}$ (которое зависит только от гомотопического класса $[\gamma]$ петли $\gamma$ ) и определяет представление $\chi$. Матрицей монодромии системы (1) в особой точке $a_{i}$ (относительно фундаментальной матрицы $\left.Y(z)\right)$ называется матрица $G_{i}$,

Работа выполнена при поддержке Российского фонда фундаментальных исследований (грант № 08-01-00342-а) и программы "Ведущие научные школы" (грант № НШ-3038.2008.1).

(С) И. В. Вьюгин, 2009 
соответствующая простой петле $\gamma_{i}$, обходящей точку $a_{i}$, т.е. $G_{i}=\chi\left(\left[\gamma_{i}\right]\right)$. При замене фундаментальной матрицы $Y(z)$ на матрицу $Y^{*}=Y(z) S$ матрица монодромии сопрягается $G_{i}^{*}=S^{-1} G_{i} S$; таким образом, система определяет представление лишь с точностью до сопряжения. Локальной монодромией будем называть произвольную матрицу, сопряженную к $G_{i}$.

Особая точка $a_{i}$ системы (1) называется фуксовой, если матрица $B(z)$ имеет простой полюс в этой точке. Фуксова особая точка линейной системы всегда является регулярной, т.е. той, где решения при приближении к особой точке внутри произвольного сектора с вершиной $a_{i}$ растут не быстрее некоторой степени $z$. Отметим, что регулярная особенность не обязана быть фуксовой (см. [1; пример 4.3]). Система (1) называется фуксовой, если все ее особые точки фуксовы.

Задача о построении фуксовой системы (1) с заданными особыми точками $a_{1}, \ldots$, $a_{n}$ и заданным представлением монодромии (2) (называемая проблемой РиманаГильберта) в общем случае имеет отрицательное решение (см. [1], [2]). Известны различные достаточные условия положительного решения этой проблемы (например, таковым является условие неприводимости представления (2)).

Предметом изучения данной работы являются фуксовы системы с вполне приводимым представлением монодромии. Основным результатом работы является следующий: любое представление ұ может быть реализовано как прямое слагаемое в представлении монодромии $\chi_{f}=\chi \oplus \tilde{\chi}$ фуксовой системы. Этот результат возник как усиление примера работы [3], давшего отрицательный ответ на следующий вопрос, поставленный Болибрухом: следует ли из разложимости (вполне приводимости) представления монодромии фуксовой системы аналогичная разложимость самой системы в прямую сумму фуксовых систем? Также этот результат является усиленным вариантом теоремы 5.3.4 работы [2].

Вторая часть работы посвящена условиям, при которых ответ на вопрос Болибруха положительный, а также их применению к построению контрпримеров к проблеме Римана-Гильберта. Доказана следующая теорема: если спектры образующих $G_{i}^{\prime}$ u $G_{i}^{\prime \prime}$ представлений $\chi_{1}$ и $\chi_{2}$ не пересекаются ни в одной точке $a_{i}, i=1, \ldots, n$, то из того, что $\chi=\chi_{1} \oplus \chi_{2}-$ представление монодромии фуксовой системы, следует, что представления $\chi_{1}$ и $\chi_{2}$ могут быть реализованы как представления монодромии фуксовых систем. На основе этого результата предложен наиболее простой из существующих способ построения серий контрпримеров к проблеме Римана-Гильберта на основе любого известного контрпримера.

2. Предварительные утверждения. Все основные факты и понятия данной теории подробно описаны в [1]. Здесь мы дадим краткое их описание.

Локально, в окрестности фуксовой особой точки $a_{i}$, существует базис решений системы (1), имеющий вид

$$
Y_{i}(z)=V_{i}(z)\left(z-a_{i}\right)^{\Lambda_{i}}\left(z-a_{i}\right)^{E_{i}}
$$

с голоморфно обратимой в окрестности $a_{i}$ матрицей $V_{i}(z)$, диагональной целочисленной матрицей $\Lambda_{i}$ и матрицей

$$
E_{i}=\frac{1}{2 \pi \sqrt{-1}} \ln \widetilde{G}_{i}
$$


где собственные значения $\rho_{i}^{j}$ матрицы $E_{i}$ удовлетворяют условиям $\operatorname{Re} \rho_{i}^{j} \in[0,1)$, a $\widetilde{G}_{i}$ - матрица локальной монодромии, т.е. $\widetilde{G}_{i}=S^{-1} G_{i} S$. Если при этом матрица (5) имеет полюс первого порядка, то этот базис называется ассоииированным. В случае, когда диагональные элементы $\Lambda_{i}$ убывают, а $E_{i}$ - верхнетреугольная, базис называется левелевским. Базис, полученный объединением левелевских базисов собственных подпространств, называется слабо левелевским. В [1; лекция 5] показано, что левелевский и слабо левелевский базисы существуют.

Метод решения задач, связанных с проблемой Римана-Гильберта, был развит Болибрухом и состоит в следующем: по представлению (2) на сфере Римана строится семейство $\mathscr{F}$ голоморфных векторных расслоений ранга $p$ с логарифмическими связностями, имеющими данные особые точки и данную монодромию. Напомним, что расслоение $F$ ранга $p$ задается набором $\left\{U_{i}\right\}$ окрестностей, покрывающих сферу, и коциклом - набором $\left\{g_{i j}\right\}$ функций склейки - голоморфно обратимых матриц размера $p$, определенных на непустых пересечениях $U_{i} \cap U_{j} \neq \varnothing$ (т.е. $g_{i j}(z)$ голоморфна в $U_{i} \cap U_{j}$ и $\operatorname{det} g_{i j}(z)$ не обращается там в нуль) и обладающих следующими свойствами:

1) $g_{i j}(z)=g_{j i}^{-1}(z)$;

2) $g_{i j}(z) g_{j k}(z) g_{k i}(z) \equiv I$, если $U_{i} \cap U_{j} \cap U_{k} \neq \varnothing$.

Связность $\nabla$ задается набором $\left\{\omega^{i}\right\}$ локальных матричных дифференциальных 1-форм ( $\omega^{i}$ определена в $\left.U_{i}\right)$ на пересечениях $U_{i} \cap U_{j} \neq \varnothing$, удовлетворяющих условиям склейки:

$$
\omega^{i}=\left(d g_{i j}\right) g_{i j}^{-1}+g_{i j} \omega^{j} g_{i j}^{-1} .
$$

Локально связность определяет систему $d y=\omega^{i} y$. Монодромия связности (так же как и монодромия системы (1)) описывает характер ветвления решений этих локальных систем при аналитическом продолжении вдоль замкнутых путей, обходящих особые точки. Эти решения называют горизонтальными сечениями связности. Связность $\nabla$ называется логарифмической (фуксовой), если все особые точки форм $\omega^{i}$ суть полюса первого порядка. Проблема Римана-Гильберта для данного представления (2) решается положительно, если окажется, что одно из расслоений семейства $\mathscr{F}$ голоморфно тривиально. Тогда в качестве коциклов $g_{i j}$ можно выбрать единичные матрицы и в силу условия склейки (4) связность $\nabla$ будет задавать фуксову систему с монодромией (2), определенную на всей сфере Римана.

Каждое расслоение $F^{\Lambda, S}$ задается набором $\Lambda=\left\{\Lambda_{1}, \ldots, \Lambda_{n}\right\}$ допустимых мат$p u и, \Lambda_{i}$ (удовлетворяющих тем же, что в (3) условиям), набором матриц приведения $S=\left\{S_{1}, \ldots, S_{n}\right\}$ и имеет следующее координатное описание: сфера покрывается набором $O_{1}, \ldots, O_{n}$ малых окрестностей точек $a_{1}, \ldots, a_{n}$ и набором $\left\{U_{\alpha}\right\}$, дополняющим набор $\left\{O_{i}\right\}$. Для каждого непустого пересечения $O_{i} \cap U_{\alpha}$ склеивающий коцикл $g_{i \alpha}(z)$ записывается в виде

$$
g_{i \alpha}(z)=\left(z-a_{i}\right)^{\Lambda_{i}}\left(z-a_{i}\right)^{E_{i}} S_{i}^{-1}
$$

где

$$
E_{i}=\frac{1}{2 \pi i} \ln \left(S_{i}^{-1} G_{i} S_{i}\right)
$$

и $\Lambda_{i}$ имеют тот же вид, что в $(3)$.

Для непустых пересечений $O_{i} \cap U_{\alpha} \cap U_{\beta}$ коцикл $g_{i \beta}(z)$ является аналитическим продолжением коцикла $g_{i \alpha}(z)$ и $g_{\alpha \beta}(z) \equiv$ const. 
Связность $\nabla^{\Lambda}$ задается нулевыми в $U_{\alpha}$ формами $\omega^{\alpha}$ и формами $\omega^{\Lambda_{i}}$, имеющими в окрестностях $O_{i}$ вид

$$
\omega^{\Lambda_{i}}=\left(\Lambda_{i}+\left(z-a_{i}\right)^{\Lambda_{i}} E_{i}\left(z-a_{i}\right)^{-\Lambda_{i}}\right) \frac{d z}{z-a_{i}}
$$

(из определения допустимой матрицы $\Lambda_{i}$ и верхнетреугольности матрицы $E_{i}$ следует, что $z=a_{i}-$ полюс первого порядка формы $\left.\omega^{\Lambda_{i}}\right)$. Нетрудно проверить, что на непустых пересечениях $O_{i} \cap U_{\alpha}$ и $U_{\alpha} \cap U_{\beta}$ формы $\omega^{\Lambda_{i}}, \omega^{\alpha}$ и $\omega^{\alpha}, \omega^{\beta}$ удовлетворяют условиям склейки (4).

Теорема Биркгофа-Гротендика утверждает, что любое голоморфное векторное расслоение $F$ ранга $p$ на сфере Римана эквивалентно расслоению, имеющему следующее описание:

$$
U_{0}=\mathbb{C}, \quad U_{\infty}=\overline{\mathbb{C}} \backslash\{0\}, \quad g_{0 \infty}=z^{K}, \quad K=\operatorname{diag}\left(k_{1}, \ldots, k_{p}\right),
$$

где $k_{1} \geqslant \cdots \geqslant k_{p}$ - набор целых чисел, который называется типом расщепления расслоения $F$.

Рассмотрим семейство $\mathscr{F}$ голоморфных векторных расслоений $F^{\Lambda, S}$ на сфере Римана с логарифмическими связностями $\nabla^{\Lambda, S}$, имеющими заданную монодромию (2).

Собственные значения $\beta_{i}^{j}=\lambda_{i}^{j}+\rho_{i}^{j}$ матрицы $\Lambda_{i}+E_{i}$ называются показателями связности $\nabla^{\Lambda, S}$ в точке $z=a_{i}$. Из вида (5) формы связности $\omega^{\Lambda_{i}, S_{i}}$ следует, что показатели в фуксовой точке $z=a_{i}$ - это собственные значения матрицы-вычета $\operatorname{res}_{a_{i}} \omega^{\Lambda_{i}, S_{i}}$.

Логарифмическая связность в тривиальном расслоении задает фуксову систему.

ОПРедЕЛЕниЕ 1. Степенъю $\operatorname{deg} F^{\Lambda, S}$ расслоения $F^{\Lambda, S}$ называется число

$$
\operatorname{deg} F^{\Lambda, S}=\sum_{i=1}^{n} \operatorname{res}_{a_{i}} \operatorname{tr} \omega^{\Lambda_{i}}=\sum_{i=1}^{n} \sum_{j=1}^{p} \beta_{i}^{j}=\operatorname{tr} K .
$$

Наклоном расслоения называется отношение его степени к рангу $\mu(F)=\operatorname{deg} F / \operatorname{rk} F$.

ОПРЕДЕЛЕНИЕ 2. Стабилъной (соответственно полустабилъной) парой называется расслоение со связностью $(F, \nabla)$ такое, что для любого инвариантного относительно связности подрасслоения $F_{\text {sub }}$ расслоения $F$ выполняется неравенство

$$
\mu\left(F_{\mathrm{sub}}\right)<\mu(F) \quad\left(\text { соответственно } \quad \mu\left(F_{\mathrm{sub}}\right) \leqslant \mu(F)\right) .
$$

Теорема 1 (Болибрух, 2002, [4]). Если представление (2) может быть реализовано как представление монодромии логарифмической связности $\nabla$ в векторном расслоении $F$ так, что пара $\left(F^{\Lambda, S}, \nabla^{\Lambda, S}\right)$ является стабильной, то проблема Римана-Гильберта для этого представления имеет положительное решение, т.е. представление (2) является представлением монодромии некоторой фуксовой системь.

\section{3. Фуксовы системы с вполне приводимой монодромией.}

Теорема 2. Любое представление х может быть реализовано как прямое слагаемое в представлении монодромии фуксовой системы $\chi_{f}=\chi \oplus \tilde{\chi}$. 
ДоКАЗАТЕЛЬСТво. Вопрос в том, чтобы для произвольного представления $\chi$ найти такое представление $\widetilde{\chi}$, что их прямая сумма $\chi_{f}$ может быть реализована как представление монодромии фуксовой системы. Интересен, конечно, только тот случай, когда $\chi$ не реализуется как монодромия фуксовой системы. Согласно теореме 1 для того, чтобы доказать, что $\chi_{f}$ может быть реализовано как монодромия фуксовой системы, достаточно показать, что существует стабильное расслоение $F^{\Lambda, S}$ с логарифмической связностью $\nabla^{\Lambda, S}$ (стабильная пара), имеющей монодромию $\chi_{f}$. Это условие является достаточным условием положительной разрешимости проблемы Римана-Гильберта (см. теорему 1).

Теперь перейдем к построению представления $\widetilde{\chi}$. Пусть представление $\chi$ имеет образующие $G_{1}, \ldots, G_{n}$ в точках $a_{1}, \ldots, a_{n}$ соответственно. Дополнительно потребуем, чтобы матрица $G_{1}$ имела жорданову нормальную форму, это всегда можно предполагать, так как представление монодромии определено с точностью до сопряжения на постоянную невырожденную матрицу. Представление $\widetilde{\chi}$ задается матрицами $\widetilde{G}_{1}, \ldots, \widetilde{G}_{n}$, которые нужно определить. Определим их следующими условиями:

(a) $\widetilde{G}_{1}=G_{1}$;

(б) матрица $\widetilde{G}_{2}$ такая, что ни одно из ее собственных значений не совпадает с собственными значениями $G_{2}$;

(в) набор матриц $\widetilde{G}_{1}, \ldots, \widetilde{G}_{n}$ определяет неприводимое представление $\widetilde{\chi}$. Сначала мы докажем, что при любом $\chi$ существуют представления $\tilde{\chi}$, удовлетворяющие этим трем условиям. Далее мы докажем, что удовлетворяющее таким условиям представление $\chi_{f}=\chi \oplus \widetilde{\chi}$ должно реализовываться как монодромия фуксовой системы.

Сначала докажем, что такие представления существуют. Покажем, что размерность множества представлений $\{\widehat{\chi}\}$, удовлетворяющих условию (а), но не удовлетворяющих условиям (б) и (в), меньше, чем размерность множества всех представлений, удовлетворяющих (а). Из этого будет следовать существование представлений удовлетворяющих условиям (a), (б) и (в) одновременно. Заметим, что выбрав только две матрицы $\widetilde{G}_{1}$ и $\widetilde{G}_{2}$, можно обеспечить выполнение необходимых условий. Остальные матрицы могут быть выбраны произвольным образом, лишь с обязательным условием $\widetilde{G}_{1} \cdots \widetilde{G}_{n}=I$. Матрица $\widetilde{G}_{1}$ определена условием (а), а наложенные на матрицу $\widetilde{G}_{2}$ ограничения не уменьшают размерность множества подходящих матриц. Невыполнение условий (б) и (в) задает множество положительной коразмерности. Действительно, множество матриц, имеющих хотя бы одно собственное значение, совпадающее с каким-нибудь собственным значением матрицы $G_{2}$, имеет положительную коразмерность 1. Аналогично и с другим условием: множество матриц $\widetilde{G}_{2}$ таких, что набор из двух матриц $\widetilde{G}_{1}, \widetilde{G}_{2}$ может быть одновременно приведен к блочно-верхнетреугольному виду, имеет положительную коразмерность для любой заданной условием (а) матрицы $\widetilde{G}_{1}$. Исключением является лишь случай $\widetilde{G}_{1}=\lambda I$, но этот случай удовлетворяет условиям теоремы Племеля (см. теорему 10.2 из [1]), которая утверждает, что представление с хотя бы одной диагональной образующей может быть реализовано как представление монодромии фуксовой системы. Следовательно, этот случай не интересен. Так из соображений размерности получаем, что почти все представления с условием (а) удовлетворяют условиям (б) и (в).

Доказывать, что можно построить фуксову систему с монодромией $\chi_{f}$, мы будем с помощью построения стабильной пары $\left(F^{\Lambda, S}, \nabla^{\Lambda, S}\right)$. Расслоение со связностью 
определим с помощью координатного описания, как это объяснено в п. 2. Здесь нам потребуется следующая техническая лемма, дающая связь между ассоциированными в различных точках базисами горизонтальных сечений связности, заданной формами (5).

Лемма 1. Рассмотрим построенное в $n .2$ расслоение со связностъю $\left(F^{\Lambda, S}, \nabla^{\Lambda, S}\right)$. Тогда ассочиированные базисъ, имеющие в $a_{i}$ вид

$$
Y_{i}(z)=\left(z-a_{i}\right)^{\Lambda_{i}}\left(z-a_{i}\right)^{E_{i}}, \quad E_{i}=\frac{1}{2 \pi i} \ln S_{i}^{-1} G_{i} S_{i},
$$

аналитически продолженные вдоль отмеченных (тех, по которым строилосъ расслоение в п.2) путей в исходную точку $z_{0}$, имеют вид $Y_{i}\left(z_{0}\right)=S_{i}$.

ДокАЗАТЕЛЬСтво. Продолжим аналитически базис $Y_{i}=\left(z-a_{i}\right)^{\Lambda_{i}}\left(z-a_{i}\right)^{E_{i}}$ как базис горизонтальных сечений связности вдоль отмеченного пути из окрестности $U_{i}$ особой точки $a_{i}$ в точку $z_{0}$. В результате аналитического продолжения получим

$$
Y_{i}\left(z_{0}\right)=g_{\alpha i} Y_{i}=S_{i}\left(z-a_{i}\right)^{-E_{i}}\left(z-a_{i}\right)^{-\Lambda_{i}}\left(z-a_{i}\right)^{\Lambda_{i}}\left(z-a_{i}\right)^{E_{i}}=S_{i},
$$

где индекс $\alpha$ указывает на окрестность $U_{\alpha}$, которая содержит $a_{i}$ на границе. Заметим, что при аналитическом продолжении из окрестности $U_{\alpha}$ в $z_{0}$ вдоль отмеченных путей базис не изменился, это следует из построения расслоения.

Далее, рассмотрим представление $\chi_{f}=\chi \oplus \tilde{\chi}$. Для того, чтобы задать пару (расслоение со связностью) $\left(E_{f}, \nabla_{f}\right)$, необходимо определить наборы матриц $\Lambda=\left\{\Lambda_{1}, \ldots, \Lambda_{n}\right\}$ и $S=\left\{S_{1}, \ldots, S_{n}\right\}$. Определим матрицы приведения следующим образом:

$$
S_{1}=\left(\begin{array}{ll}
I & 0 \\
I & I
\end{array}\right)
$$

где $I$ - $p$-мерный единичный блок, а остальные матрицы $S_{2}, \ldots, S_{n}$ выберем произвольным допустимым способом, например, как матрицы приведения матриц $G_{2}, \ldots$, $G_{n}$ к жордановой нормальной форме. Матрицы нормирования возьмем

$$
\Lambda_{1}=\left(\begin{array}{cc}
d \cdot I & 0 \\
0 & 0
\end{array}\right), \quad \Lambda_{2}=\cdots=\Lambda_{n}=0,
$$

где $d$ - достаточно большое натуральное число. Теперь расслоение со связностью определено, и осталось доказать лишь, что пара $\left(E_{f}, \nabla_{f}\right)$ стабильна.

Установим, какие имеются инвариантные подпространства, т.е. какие у $\chi_{f}$ есть подпредставления. Покажем, что кроме подпредставлений $\chi_{\alpha}$ представления $\chi$, а также их прямых сумм $\chi_{\alpha} \oplus \tilde{\chi}$, в том числе самих представлений $\chi$ и $\widetilde{\chi}$, никаких других подпредставлений у $\chi_{f}$ нет. Глядя на образующую монодромии $\chi_{f}$ в точке $a_{2} G_{2}^{f}=G_{2} \oplus \widetilde{G}_{2}$, видим, что любое инвариантное подпространство этого оператора распадается в прямую сумму инвариантных подпространств оператора $G_{2}$ и оператора $\widetilde{G}_{2}$, которые не пересекаются (имеют нулевое пересечение), так как матрицы $G_{2}$ и $\widetilde{G}_{2}$ по построению не имеют одинаковых собственных значений. Тогда видим, что мы перебрали все подпредставления, так как $\widetilde{\chi}$ неприводимо.

Теперь нужно показать, что выполняется условие стабильности, т.е., что это условие выполнено для любого подрасслоения $F_{\text {sub }}$ из следующих четырех возможных 
типов $F_{\chi}, F_{\chi_{\alpha}}, F_{\chi_{\alpha} \oplus \tilde{\chi}}$ или $F_{\tilde{\chi}}$, где нижний индекс обозначает подпредставление представления $\chi_{f}$, которому соответствует подрасслоение. Только это подрасслоение стабилизируется связностью $\nabla$. Покажем, что выполнено неравенство

$$
\mu\left(F_{\text {sub }}\right)<\mu(F), \quad F=F_{\chi_{f}} .
$$

Так как расслоение со связностью определено, мы можем явно вычислить степени всех подрасслоений. Так как мы явно не определяли представление, мы не знаем собственных значений образующих монодромии, от которых зависят дробные части асимптотик, а как известно, сумма асимптотик равна степени. Но вклад дробных частей ограничен, и поэтому мы получим ограничения на степени и не будем выражать их точно через неизвестные нам собственные значения операторов $\chi_{f}$. Имеем

$$
\operatorname{deg}(F) \in[d p, d p+2 n p), \quad \mu(F) \in\left[\frac{d}{2}, \frac{d}{2}+n\right) .
$$

Обозначим размерность $\operatorname{dim} \chi_{\alpha}=k, k<p$. Тогда имеем следующие соотношения на степени и наклоны:

a) $\operatorname{deg} F_{\chi} \in[0, n p), \quad \mu\left(F_{\chi}\right) \in[0, n)$;

б) $\operatorname{deg}\left(F_{\chi_{\alpha}}\right) \in[0,2 n k), \mu\left(F_{\chi_{\alpha}}\right) \in[0, n)$;

в) $\operatorname{deg}\left(F_{\chi_{\alpha} \oplus \tilde{\chi}}\right) \in[d k, d k+(k+p) n), \mu\left(F_{\chi_{\alpha} \oplus \tilde{\chi}}\right) \in[d(1-p /(k+p)), d(1-p /(k+p))+n)$;

г) $\operatorname{deg}\left(F_{\tilde{\chi}}\right) \in[0, n p), \mu\left(F_{\tilde{\chi}}\right) \in[0, n)$.

Видно, что наклон расслоения $F$ не меньше, чем $d / 2$, а наклоны подрасслоений не превосходят величины $d(p-1) /(2 p-1)+n$, которая достигается при $\operatorname{dim} \chi_{\alpha}=p-1$. Тогда при $d>4 p n$ условие стабильности выполнено.

\section{4. Контрпримеры к проблеме Римана-Гильберта.}

ТеОРема 3. Если спектры образующих представлений $\chi_{1}$ и $\chi_{2}$ не пересекаются ни в одной точке, то из того, что $\chi=\chi_{1} \oplus \chi_{2}$ - представление монодромии фуксовой системь, следует, что и представления $\chi_{1}$ и $\chi_{2}$ могут быть реализованы как монодромии фуксовых систем.

ДокАЗАТЕЛЬСтво. Действительно, пусть задана система с монодромией $\chi$, тогда эта система определяет логарифмическую связность $\nabla$ в тривиальном расслоении $F$. Поскольку расслоение тривиально, то оно со связностью образует стабильную или полустабильную пару, так как тривиальное расслоение само полустабильно. Подпредставлениям $\chi_{1}$ и $\chi_{2}$ отвечают подрасслоения $F_{1}$ и $F_{2}$ соответственно. Так как пара $(F, \nabla)$ полустабильна и степени нуль, подрасслоения $F_{1}$ и $F_{2}$ имеют неположительную степень, т.е. $\operatorname{deg} F_{1} \leqslant 0, \operatorname{deg} F_{2} \leqslant 0$. В качестве ассоциированного базиса рассмотрим слабо левелевский. Инвариантные подпространства, отвечающие прямым слагаемым монодромии, порождаются частями слабо левелевского базиса, т.е. первые $l$ векторов порождают первое инвариантное подпространство, а последние $p-l$ векторов - второе. Действительно, рассмотрим оператор $G_{i}=G_{i}^{\prime} \oplus G_{i}^{\prime \prime}$. По условию, операторы $G_{i}^{\prime}$ и $G_{i}^{\prime \prime}$ не имеют одинаковых собственных значений, а значит, каждый вектор из слабо левелевского базиса лежит ровно в одном из подпространств в том, в котором лежит соответствующее собственное подпространство. Тогда степень подрасслоения $F_{1}$ равна сумме показателей, соответствующих подпредставлению $\chi_{1}: \operatorname{deg} F_{1}=\sum_{i=1}^{n} \sum_{j=1}^{l} \beta_{i}^{j}$, то же верно и для $F_{2}$, т.е. $\operatorname{deg} F_{1}=\sum_{i=1}^{n} \sum_{j=l+1}^{p} \beta_{i}^{j}$. 
Следовательно, $\operatorname{deg} F=\sum_{i=1}^{n} \sum_{j=1}^{p} \beta_{i}^{j}$ и $\operatorname{deg} F=\operatorname{deg} F_{1}+\operatorname{deg} F_{2}$. Отсюда следует, что $\operatorname{deg} F_{1}=0$ и $\operatorname{deg} F_{2}=0$, т.е. $F_{1}$ и $F_{2}$ - подрасслоения тривиального расслоения $F$, имеющие степень нуль, но тогда они тоже тривиальны (см. [1]). Следовательно, связности в подрасслоениях $F_{1}$ и $F_{2}$ представляют собой фуксовы системы.

Теперь с помощью полученного достаточного условия приведем метод построения новой серии контрпримеров к проблеме Римана-Гильберта. Болибрухом был построен следующий контрпример для случая трех особых точек в размерности четыре (пример 11.1 из [1]):

$$
\begin{aligned}
& G_{1}=\left(\begin{array}{cccc}
1 & 1 & 0 & 0 \\
0 & 1 & 1 & 0 \\
0 & 0 & 1 & 1 \\
0 & 0 & 0 & 1
\end{array}\right), \quad G_{2}=\left(\begin{array}{cccc}
3 & 1 & 1 & -1 \\
-4 & -1 & 1 & 2 \\
0 & 0 & 3 & 1 \\
0 & 0 & -4 & -1
\end{array}\right), \quad G_{3}=\left(\begin{array}{cccc}
-1 & 0 & 2 & -1 \\
4 & -1 & 0 & 1 \\
0 & 0 & -1 & 0 \\
0 & 0 & 4 & -1
\end{array}\right) \\
& S_{2}^{-1} G_{2} S_{2}=\left(\begin{array}{cccc}
1 & 1 & 0 & 0 \\
0 & 1 & 1 & 0 \\
0 & 0 & 1 & 1 \\
0 & 0 & 0 & 1
\end{array}\right), \quad S_{2}=\frac{1}{3}\left(\begin{array}{cccc}
3 & 0 & 0 & 0 \\
-6 & 3 & -3 & 4 \\
0 & 0 & 1 & -1 \\
0 & 0 & -2 & 3
\end{array}\right) \\
& S_{3}^{-1} G_{3} S_{3}=\left(\begin{array}{cccc}
-1 & 1 & 0 & 0 \\
0 & -1 & 1 & 0 \\
0 & 0 & -1 & 1 \\
0 & 0 & 0 & -1
\end{array}\right), \quad S_{3}=\frac{1}{64}\left(\begin{array}{cccc}
0 & 16 & 4 & 3 \\
64 & 0 & 0 & 0 \\
0 & 0 & 0 & -4 \\
0 & 0 & -16 & -12
\end{array}\right)
\end{aligned}
$$

В [1] показано, что контрпримерами к проблеме Римана-Гильберта являются все Б-представления, т.е. приводимые представления, образующие которых приводятся к жордановой клетке с собственными значениями, произведение которых по всем точкам не равно 1 . Здесь это $1 \times 1 \times(-1)=-1 \neq 1$.

Теорема 4. Существуют контрпримеры к проблеме Римана-Гилъберта в размерности р с п особыми точками для всех пар $(p, n)$ с $p \geqslant 4, n \geqslant 3$.

На самом деле, примеры существуют также и для $p \geqslant 3, n \geqslant 4$. Контрпримеры во всех размерностях построены в [2], [5], но мы приводим другие, значительно более простые, серии примеров.

ДокАЗАТЕЛЬСтво. Построим примеры в размерности $p=4$ и для произвольного числа особых точек $n \geqslant 3$. Рассмотрим матрицу $G_{1}$ : она - жорданова клетка с собственным значением 1 . Заметим, что ее степень $G_{1}^{n-2}$ тоже приводится к жордановой клетке с собственным значением 1 , так как ранг матрицы $\left(G_{1}^{n-2}-I\right)$ очевидно равен трем. Пусть $S_{1}^{\prime}$ - матрица приведения, т.е. $S_{1}^{\prime-1} G_{1}^{n-2} S_{1}^{\prime}=G_{1}$. Таким образом, представление $\tilde{\chi}$ с образующими

$$
\widetilde{G}_{1}=S_{1}^{\prime-1} G_{1} S_{1}^{\prime}, \quad \ldots, \quad \widetilde{G}_{n-2}=S_{1}^{\prime-1} G_{1} S_{1}^{\prime}, \quad \widetilde{G}_{n-1}=G_{2}, \quad \widetilde{G}_{n}=G_{3},
$$

является Б-представлением, произведение собственных значений которого по всем точкам также равно -1. Следовательно, $\tilde{\chi}$ является контрпримером к проблеме Римана-Гильберта.

Вторым шагом распространим контрпримеры на любую размерность. Рассмотрим представление $\chi_{e}$ со скалярными образующими

$$
G_{i}^{e}=c_{i} I, \quad i=1, \ldots, n,
$$


с $c_{1} \cdots c_{n}=1$ и $c_{i} \neq 1,-1, i=1, \ldots, n$. Тогда, если размерность $\chi_{e}$ равна $p-4$, то представление $\chi=\chi_{e} \oplus \widetilde{\chi}$ по теореме 3 и по только что доказанному является контрпримером к проблеме Римана-Гильберта в размерности $p$ с $n$ особыми точками.

В заключение хочу выразить благодарность В. П. Лексину и Р. Р. Гонцову за внимание к работе и за существенные замечания.

\section{СПИСОК ЦИТИРОВАННОЙ ЛИТЕРАТУРЫ}

[1] А.А. Болибрух, Фуксовы дифференциальные уравнения и голоморфные расслоения, Современные лекционные курсы, МЦНМО, М., 2000.

[2] А. А. Болибрух, 21-я проблема Гильберта для линейных фуксовых систем, Тр. МИАН, 206, Наука, М., 1994.

[3] И.В. Вьюгин, "Неразложимая фуксова система с разложимым представлением монодромии", Матем. заметки, 80:4 (2006), 501-508.

[4] А. А. Болибрух, "Проблема Римана-Гильберта на компактной римановой поверхности", Монодромия в задачах алгебрачческой геометрии и дифференииальных уравнений, Тр. МИАН, 238, Наука, М., 2002, 55-69.

[5] H. Esnault, C. Hertling, Semistable Bundles on Curves and Reducible Representation of the Fundamental Group, arXiv: math.AG/0101194.

И. В. Вьюгин

Московский государственный университет им. М. В. Ломоносова
Поступило 20.06.2008 